\title{
FDA Center For Devices and Radiological Health Terminology
}

National Cancer Institute

\section{Source}

National Cancer Institute. FDA Center For Devices and Radiological Health Terminology. NCI Thesaurus. Code C62596.

Terminology used in individual case reports for voluntary and mandatory reporting of adverse reactions or adverse events during both pre and post approval periods of medical device development and usage. 